\title{
Improved Long-Term Reliability of a Silica-Encapsulated Perovskite Quantum-Dot Light-Emitting Device with an Optically Pumped Remote Film Package
}

Shun-Chieh Hsu, Yu-Ming Huang, Chung-Ping Huang, Ting-Yu Lee, Yu-Yun Cho, Yin-Hsin Liu, Arumugam Manikandan, Yu-Lun Chueh, Teng-Ming Chen, Hao-Chung Kuo, and Chien-Chung Lin*

Cite This: ACS Omega 2021, 6, 2836-2845

Read Online

ABSTRACT: In this study, inorganic perovskite $\left(\mathrm{CsPbBr}_{3}\right)$ quantum dots are wrapped in $\mathrm{SiO}_{2}$ to provide better performance against external erosion. Long-term storage ( 250 days) is demonstrated with very little changes in the illumination capability of these quantum dots. While in the continuous aging procedure, different package architectures can achieve very different lifetimes. As long as $6000 \mathrm{~h}$ of lifetime can be expected from these quantum dots, but the blue shift of emission wavelength still needs more investigation.

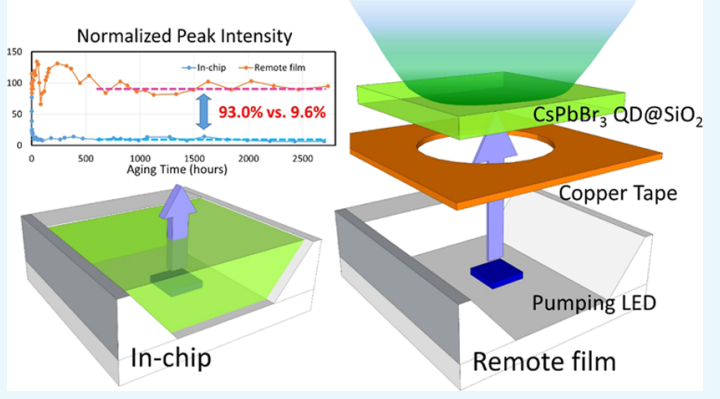

\section{INTRODUCTION}

Since its first introduction in the past decade, halide perovskite-related materials have gathered intense attention due to their high efficiencies in illumination and photoabsorption. ${ }^{1,2}$ Highly efficient perovskite solar cells have achieved $25.1 \%$ of power conversion efficiency recently, ${ }^{3}$ and the extraordinary light-emitting capability of the perovskite material cannot be overlooked when the candidate for the next-generation light source is considered. While the original organic halide composites $\left(\mathrm{CH}_{3} \mathrm{NH}_{3} \mathrm{PbX}_{3}, \mathrm{X}=\mathrm{I}, \mathrm{Br}, \mathrm{Cl}\right)$ are effective in providing different colors, a new breed of inorganic metal halide materials, such as $\mathrm{CsPbX}_{3}$, where $\mathrm{X}=\mathrm{I}, \mathrm{Br}, \mathrm{Cl}$, poses a new opportunity for the device. ${ }^{4}$ In addition to different colors provided by different compositions, it is also possible to exploit the quantum confinement effect on these materials to realize the tunability in color via different sizes of nanoparticles of metal halide perovskites. ${ }^{4}$ Furthermore, the color purity provided by perovskite quantum dots (PQDs) also prompts a much wider color gamut coverage on the CIE color space, which is greatly desired for the next-generation display. ${ }^{4-6}$ However, they are very sensitive to environmental factors like temperature, oxygen, light, water, etc., and various methods have been implemented to extend the lifetime of the QDs. One of the most common methods is to coat these PQDs with a protective layer that is made of either polymer, silica, or other substances. ${ }^{7-11}$ Among these methods, coating the QDs with $\mathrm{SiO}_{2}$ is one of the potential choices. The coating process can be integrated into the synthesis procedure, and the protection effectiveness of this $\mathrm{SiO}_{2}$ material is well known in the semiconductor industry. At the same time, the silica shell can also prevent anion exchange if a further mixture of different species of perovskite QDs is necessary. ${ }^{12,13}$ In this paper, the cesium lead tribromide $\left(\mathrm{CsPbBr}_{3}\right)$ perovskite quantum dots (PQDs) are coated with $\mathrm{SiO}_{2}$ and continuously tested under extreme conditions to explore the issues of stability. Different package methods are also adapted to alleviate or improve the lifetime of these QDs in this paper. The filling substances in the package can play an important role in heat dissipation, which might affect the lifetime of the PQD eventually. Some previous results have shown that hexagonal boron nitride (BN) can help to reduce the QD composite temperature, ${ }^{14}$ and we will explore the effectiveness of using boron nitride particles in this paper as well. Most importantly, the long-term evolution of photonic properties of these QDs will be reported and analyzed.

\section{RESULTS}

2.1. Performance of PQD Light-Emitting Diodes (LEDs). The first thing we should examine is the quality of the remote film and in-chip devices. The remote film was made manually in this study, and it is prone to be not as ideal as the machine-fabricated ones. First, the color of the film can be examined. From Figure 1a-c, no significant color variation can

Received: October 22, 2020

Accepted: January 8, 2021

Published: January 21, 2021 

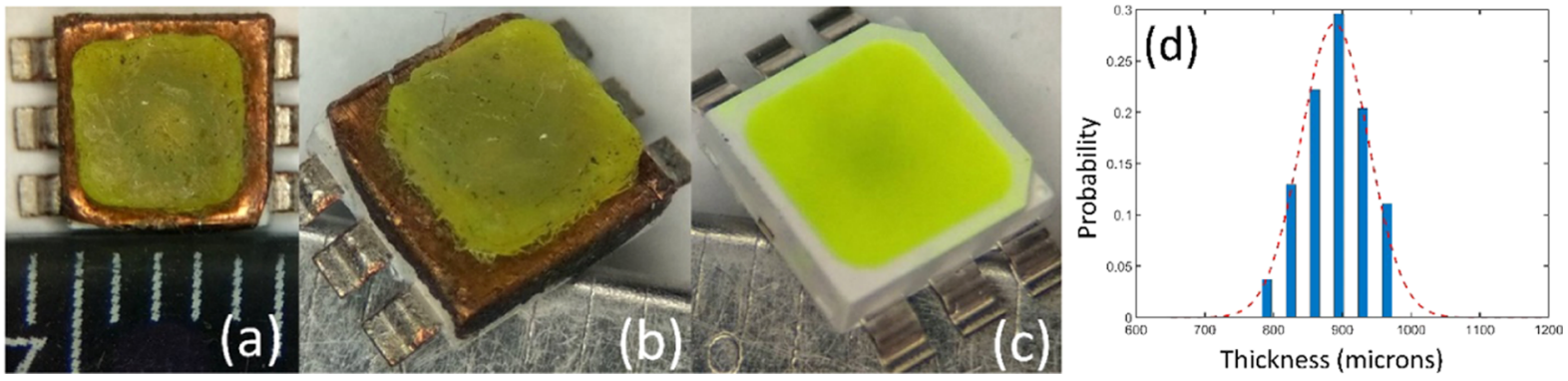

Figure 1. Device under an optical microscope: (a) top view and (b) bird's-eye view of a remote film device and (c) bird's-eye view of a fresh in-chip device. (d) Thickness histogram of the remote film. (Photograph courtesy of Yang, Jhen Jia. Copyright 2020).

be found in our PQD film, which can be regarded as a good sign of a thorough mixture of PQD and PDMS. The detailed device picture can be seen in Figure 1a,b, and the side length of the PQD@SiO ${ }_{2}$ film is around $4 \mathrm{~mm}$. In Figure 1c, a flat surface of an in-chip-type device can be observed. From the thickness measurement under an optical microscope, we found a remote film thickness distribution, like the histogram shown in Figure 1d. A Gaussian fitting can be found to perfectly match with experimental results. The mean thickness is 888.5 $\mu \mathrm{m}$, and the linewidth of the distribution is $115.5 \mu \mathrm{m}$. The picture in Figure $1 \mathrm{~b}$ indicates that the overall shape of the remote film is close to a rectangle, and the variation in thickness happens randomly across the whole film. Thus, the surface should be rough due to the manual process of its fabrication and the roughness should be close to the linewidth of distribution in Figure 1d. The controlled factors in a remote film are the quantity of the quantum dots, the total weight of the film, and the volume of the film in this study, and they can be achieved by the aforementioned molding step. Via these factors, we can control the approximate thickness of the remote films in this study and the random roughness of the QD film can facilitate the uniform scattering of incident photons. $^{15}$ We believe that the comparison in their own lifetime is valid because the aged result is compared to its own initial value, and it is the comparison of the same device that we analyzed in this paper.

Figure 2a shows the current-dependent electroluminescence (EL) spectra of a remote film device with $0.5 \mathrm{mg}$ of $\mathrm{BN}$ particles. The two-peak feature is due to the partial conversion of UV photons, and the corresponding photonic conversion efficiency (PCE) can be calculated as follows ${ }^{16,17}$
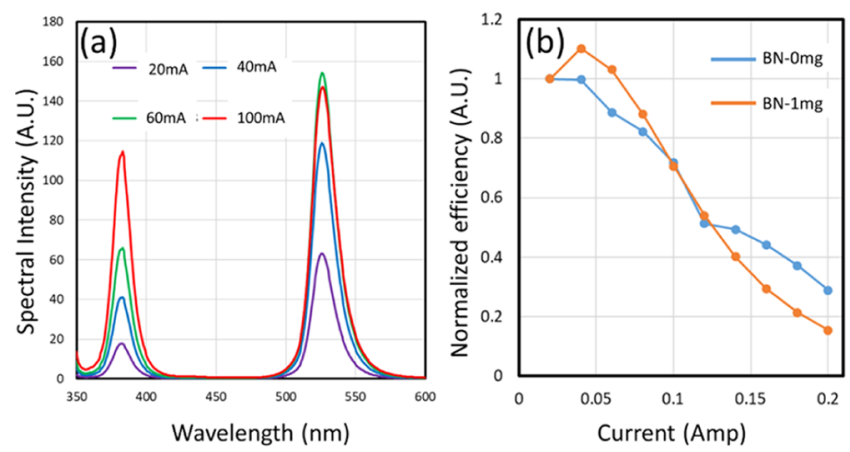

Figure 2. (a) Current-dependent spectra of a remote film device with $0.5 \mathrm{mg}$ of BN doping. (b) Normalized efficiencies of no-BN- and 1 $\mathrm{mg}$ BN-doped devices vs currents.

$$
\begin{aligned}
\text { PCE } & =\frac{\# \text { of green photons of PQD }}{\# \text { of UV photons from the LED }} \\
& =\frac{\int_{\text {green }}\left(\frac{\lambda}{h c}\right) \times\left[I_{\mathrm{em}}^{\mathrm{QD}}(\lambda)-I_{\mathrm{em}}^{\mathrm{ref}}(\lambda)\right] \mathrm{d} \lambda}{\int_{\mathrm{UV}}\left(\frac{\lambda}{h c}\right) \times\left[I_{\mathrm{ex}}^{\mathrm{ref}}(\lambda)-I_{\mathrm{ex}}^{\mathrm{QD}}(\lambda)\right] \mathrm{d} \lambda}
\end{aligned}
$$

where $\mathrm{I}_{\mathrm{ex}}^{\mathrm{QD}}$ and $I_{\mathrm{ex}}^{\mathrm{ref}}$ are the integrated intensities of the UV LED excitation source with and without the PQD layers and $I_{\mathrm{em}}^{\mathrm{QD}}$ and $I_{\mathrm{em}}^{\mathrm{ref}}$ are the intensities of the visible band (but excluding the UV band) with and without PQD layers, respectively. In this experiment, the PCE values vary among different types of packaged devices. For the in-chip type, the PCE values are around 41 and $15 \%$ for $\mathrm{BN}=0$ and $2 \mathrm{mg}$ cases, respectively. For the remote file type, the PCE is around $7.3 \%$ for the $\mathrm{BN}$ doped devices at best. The detailed information can be seen in Table 1 . The efficiency of the device generally decreases as the current increases due to the increasing temperature in the package. The current-dependent luminous efficiency can be found in the Supporting Information (Figure S1).

Another important parameter that can be extracted is the luminous efficiency $(\mathrm{lm} / \mathrm{W})$ that also takes the electrical power into account. The best result we obtained is from the in-chiptype device without any h-BN particles, and it is about $60 \mathrm{~lm} /$ $\mathrm{W}$. This value is comparable to some of the best devices reported previously. ${ }^{13}$ The corresponding efficiency in the remote film-type device is limited due to the installation of the copper tape and limited opening window on top of the lead frame. The hole at the center of the copper tape is $2.5 \mathrm{~mm}$ in diameter that can prevent some UV photons from absorbing by the PQD layer but by the copper tape. The remote film-type device with $\mathrm{BN}$ doping shows its best result in $\mathrm{BN}=0.5 \mathrm{mg}$ case, and it is around $12.5 \mathrm{~lm} / \mathrm{W}$. The device without $\mathrm{BN}$ doping still has the best efficiency of $13.48 \mathrm{~lm} / \mathrm{W}$ like their counterparts in the "in-chip" type. The BN particles cause a certain loss in efficiency as we observe the dependence of this luminous efficiency vs doping weight of h-BN particles. The role of h-BN particles is originally designed for heat dissipation, but these particles could also have unexpected optical scattering and absorption in the UV range such that the optical efficiency is eventually lowered. ${ }^{18}$ In Figure 2a, the saturation of the green peak and the increasing blue peak can be seen at high current levels, which indicates that the film becomes transparent due to overexcitation. If we normalize the luminous efficiency using low current values, we can obtain Figure $2 b$, where the low-BN-doped device can sometimes have better thermal performance than that of the no-BN sample at low to medium current. However, at higher currents, 
Table 1. Summary of Measured Parameters for All Devices

\begin{tabular}{|c|c|c|c|c|c|c|}
\hline type of package & \multicolumn{2}{|c|}{ in-chip } & \multicolumn{4}{|c|}{ remote film } \\
\hline BN weight (mg) & 0 & 2 & 0 & 0.5 & 1 & 2 \\
\hline PCE @20 mA (\%) & 41.04 & 14.94 & 8.74 & 7.29 & 2.64 & 4.63 \\
\hline luminous efficiency $(\mathrm{lm} / \mathrm{W})$ & 60.19 & 26.05 & 13.48 & 12.72 & 4.22 & 8.01 \\
\hline
\end{tabular}
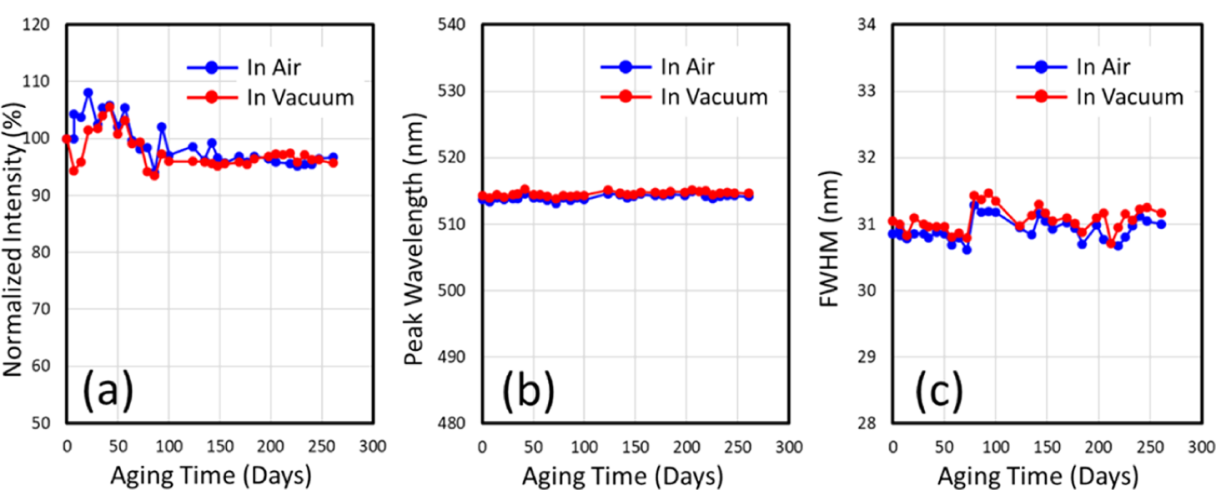

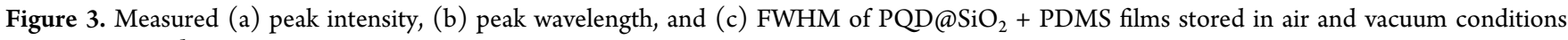
against storage days.
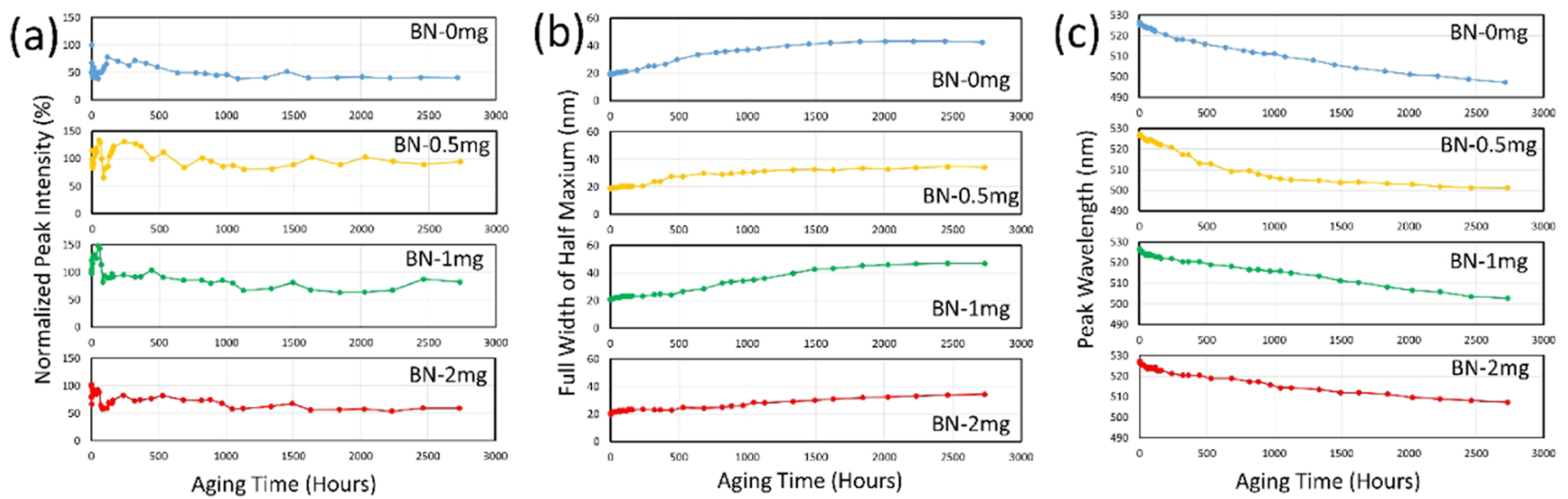

Figure 4. (a) Normalized peak intensity, (b) FWHM, and (c) peak shift of remote film samples vs aging time.

the accumulated heat due to nonradiative recombination of $\mathrm{BN}$ and PQD can really drag down the device performance.

2.2. Long-Term Aging Tests. One of the important characteristics of these $\mathrm{SiO}_{2}$-coated PQDs is the ability to withstand the erosion of external water, oxygen, and light degradation. To examine this, two samples of the PQD@SiO + PDMS mixture were placed in vacuum and in air, respectively, and they were constantly monitored by the photoluminescence (PL) system for their illumination intensities, emission peak wavelengths, and full width at halfmaxima (FWHM) of the spectra. Figure $3 a-c$ shows the results for a duration of 250 days. The PL intensities of the sample left in air are kept at the same level (95.5\% of the initial value) and show almost no changes in FWHM and emission peak wavelength in this long period (the FWHM changes for $-0.19 \mathrm{~nm}$ and the peak red-shifts for $0.60 \mathrm{~nm}$ ). The quality of the PQD@SiO 2 films is maintained very well throughout these days. It is important to acknowledge this stable long-term property of $\mathrm{PQD}$ films in the on-shelf storage condition before putting them in a harsher one.

The next thing to be verified is the long-term stability of these PQDs under continuous operations. This is an ultimate test before a novel type of LED can be commercialized.
Different from the on-shelf test, once the LED was turned on, the PQDs would be shined and heated continuously except during the measurement in the integrated sphere for monitoring. The constant photon wear-out and high-temperature environment can be really challenging toward these nanocrystals. The in-chip samples might experience the worst scenario because the direct contact to the largest heat source, i.e., the LED chip, and the poor thermal conductivity of the slurry makes the situation worse.

On the other hand, in the remote film-type device, which is similar to the remote phosphor package, ${ }^{19}$ the PQD film was suspended above the chip and contacted with copper foil for better heat conduction to alleviate the direct heating from the chip. In both types, the $\mathrm{BN}$ particles were doped into the slurry when mixing and they were expected to enhance the thermal conductivity of the overall composites.

Figure $4 a-c$ shows the over $2500 \mathrm{~h}$ of data for the remote film devices with different BN particle doping amounts, and the in-chip results can be found in the Supporting Information (Figure S2). To properly evaluate the lifetime of the devices, a criterion called LT50 can be linearly extrapolated from the last $2500 \mathrm{~h}$ of data and the degradation of the $50 \%$ initial peak intensity can be calculated. For detailed calculations, the 
formula can be found in the Supporting Information. For the in-chip-type devices, the decrease of the PQD illumination capability is dramatic. In less than $1 \mathrm{~h}$, the LT50 criterion, which is the time span for the efficiency of the device reducing to $50 \%$ of the initial value, was breached. Both the peak wavelength and FWHM trend similarly to the intensity: they both saturated quickly in several hours from the beginning of the aging process. The blue-shifted peak and widened spectrum are both observed in the samples with and without $\mathrm{BN}$ particles.

As for the remote film samples, the change is more gradual and the stabilized emission power and FWHM of the spectra can be observed after several hundreds of hours into the aging process. However, the emission peak tends to blue shift and shows no sign of stopping even after $2500 \mathrm{~h}$ of aging. Higher than $29 \mathrm{~nm}$ of blue shift can be observed from the measurement. The resulting LT50 for the remote film-type devices can be as high as $6292 \mathrm{~h}$, and all of the detailed parameters of optical spectra after the aging process can be found in Table 2.

Table 2. Measured Optical Characteristics and Lifetimes (LT50) of Various Samples

\begin{tabular}{lllllll}
\multicolumn{1}{r}{ type of package } & \multicolumn{2}{c}{ in-chip } & \multicolumn{4}{c}{ remote film } \\
BN weight (mg) & 0 & 2 & 0 & 0.5 & 1 & 2 \\
LT50 (h) & 0.32 & 0.35 & 638 & 6292 & 4549 & 2878 \\
$\lambda_{\text {Peak }}$ change (nm) & -15.0 & -19.9 & -32.6 & -25.7 & -29.4 & -21.7 \\
FWHM change & 19.6 & 19.7 & 22.9 & 17.2 & 26.4 & 14.3 \\
$\quad(\mathrm{~nm})$ & & & & & &
\end{tabular}

\section{DISCUSSION}

In addition to intensity monitoring, we also performed several material characterizations to compare between fresh and aged $\mathrm{PQD} @ \mathrm{SiO}_{2}$ powders. The purpose of this examination is to find out the variation of the PQD material during the harsh testing conditions. To prepare these samples, the PQD powders were aged (both optically and thermally) for at least $24 \mathrm{~h}$ prior to any characterization. The length of this preaging process was determined by our previous results that most of the degradation seems to happen in the first $24 \mathrm{~h}$ of the aging process.

3.1. Fourier Transform Infrared (FTIR) and X-ray Diffraction (XRD) Measurements. FTIR can provide important information of the chemical bonding in the material. In Figure 5a, both the fresh and the burnt samples were tested for FTIR. From the FTIR spectra, several featured dips can be identified from comparison to the past results: the $\mathrm{Si}-\mathrm{O}-\mathrm{Si}$ mode at around $1070 \mathrm{~cm}^{-1}$, the $\mathrm{N}-\mathrm{H}$-related bending mode at $1630 \mathrm{~cm}^{-1}$, and the mixed broadband signal at around 3000 $\mathrm{cm}^{-1}$ for $\mathrm{C}-\mathrm{H}$-related mode. ${ }^{13,20-23}$ There are no apparent differences between the burnt and fresh PQD@SiO ${ }_{2}$. The comparison shows that the compositions and bonding conditions in the PQD composites did not change before and after the aging process.

Figure $5 \mathrm{~b}$ shows the XRD comparison between the burned and fresh PQD films. The standard XRD peak locations of $\mathrm{CsPBBr}_{3}$ are also marked in the plot. The broadened peak of $\mathrm{SiO}_{2}$ can be seen at around $20^{\circ} .{ }^{13}$ The upper side of Figure $5 \mathrm{~b}$ marks the peak locations of $\mathrm{CsPbBr}_{3}, \mathrm{Cs}_{4} \mathrm{PbBr}_{6}$, and $\mathrm{PbBr}_{2}$ obtained from the Inorganic Crystal Structure Database (ICSD). Major peaks for PQDs can be observed in Figure $5 b$, such as those in $29.68,36.54,42.61^{\circ}$, etc. ${ }^{24,25}$ However, due to the presence of $\mathrm{SiO}_{2}$ with a large quantity in the sample, sometimes, it is difficult to acquire the highly resolved XRD pattern from the PQD itself. In both cases, the major peaks are kept the same in terms of peak locations and the relative intensities and linewidths. From this experiment, the change in crystallography seems to be negligible during this aging process.

3.2. Location-Dependent Photoluminescence. In Figure 6, the image of a BN-doped in-chip PQD LED after

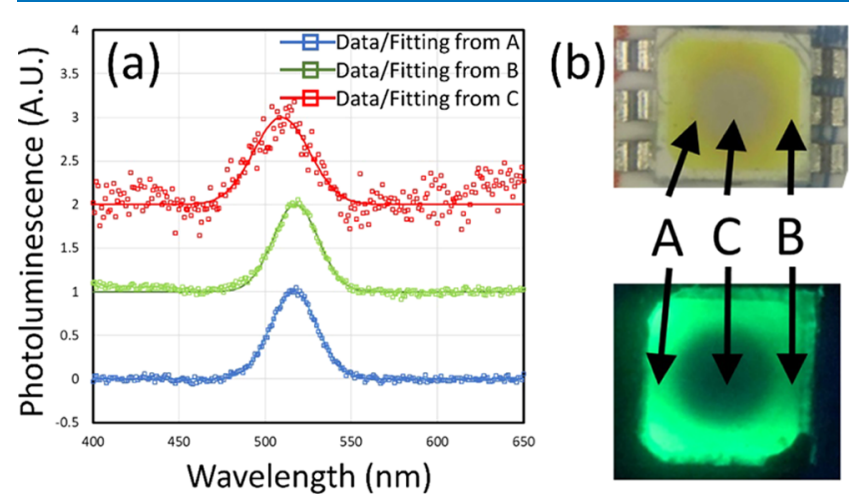

Figure 6. (a) Location-dependent PL signal of an aged in-chip device and (b) pictures of this device under natural light and UV.

the aging process is shown. A faint and circular mark can be seen at the center. This mark shows up more clearly when UV excitation illuminates upon the device (also shown in the figure). By taking the location-dependent PL, one can observe
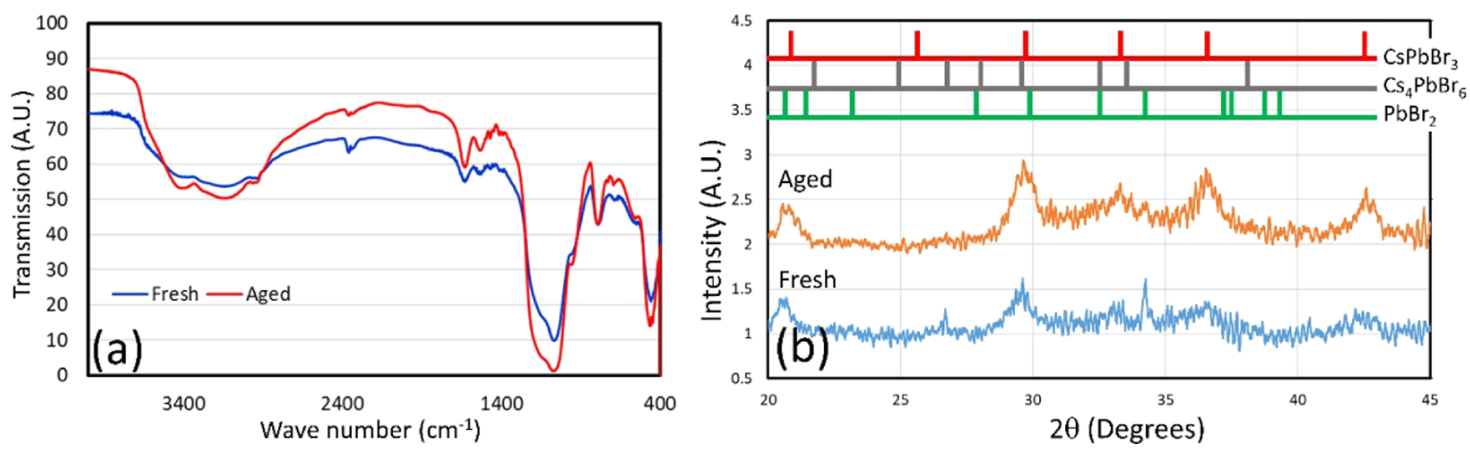

Figure 5. (a) Comparison of FTIR spectra of PQD@SiO ${ }_{2}$ powder before and after aging. (b) Comparison of the X-ray diffraction pattern of the $\mathrm{PQD} @ \mathrm{SiO}_{2}+$ PDMS film before and after aging. 

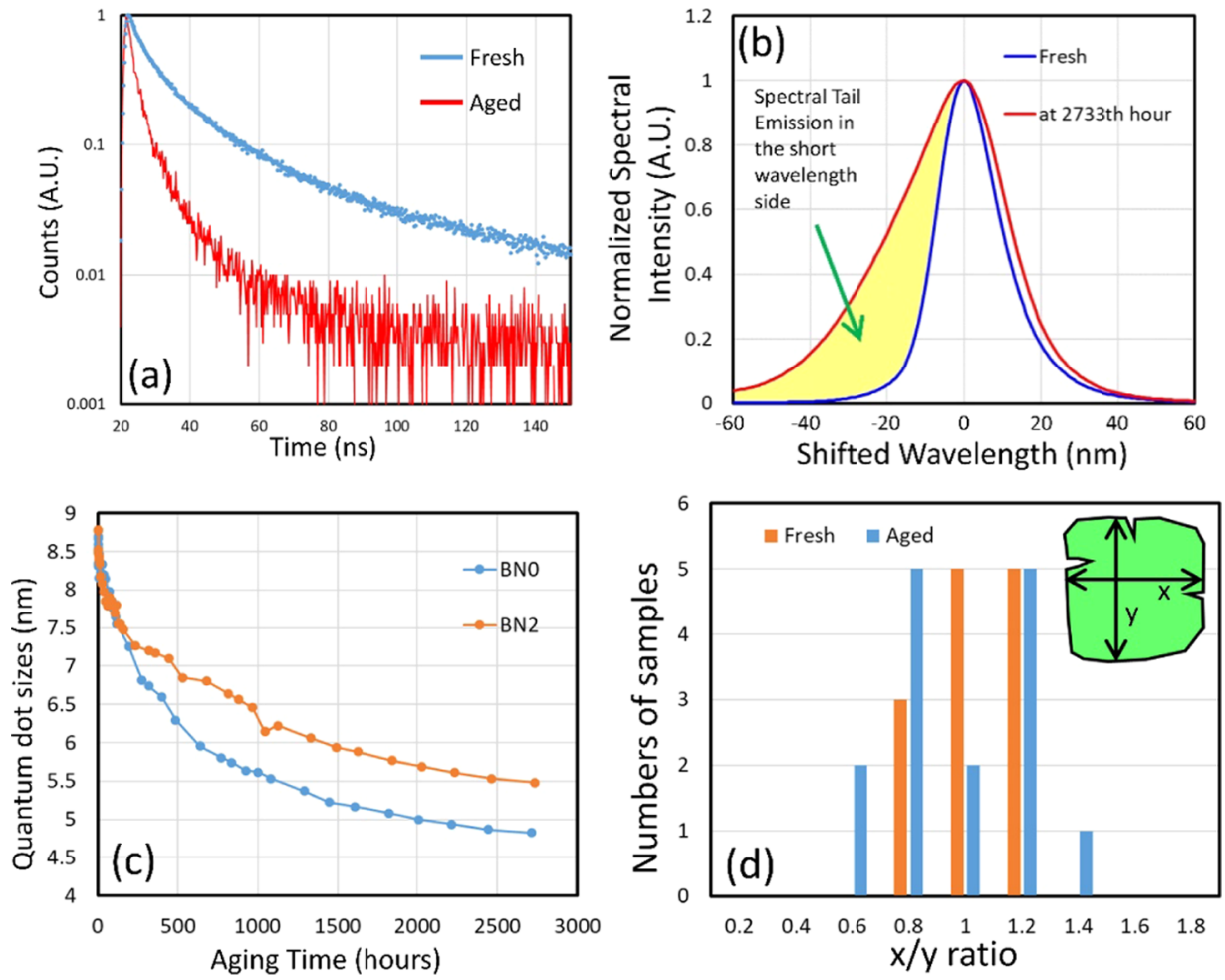

Figure 7. (a) TRPL profile of a fresh and an aged PQD sample. (b) Normalized and peak-shifted spectra overlay showing the increased tail

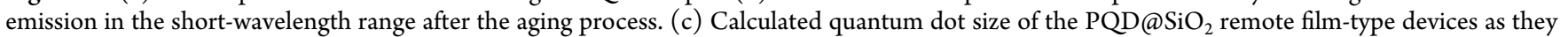
are aged over time. (d) Histogram of the dot side-length ratio of fresh and aged $\mathrm{PQD} @ \mathrm{SiO}_{2}$ powders. The inset is an illustration of the quantum dot and the definition of $x$-length and $y$-length.

the dramatic difference in the peak wavelength and the peak intensity of the specified area in Figure 6 . The central area demonstrated a six-time reduction in the peak intensity, while its peak shifts to a shorter wavelength by $7.65 \mathrm{~nm}$. The FWHM of the signal of the center is also widened by $6.74 \mathrm{~nm}$ when compared to the side area ( 31.47 and $38.21 \mathrm{~nm}$ for A and C regions, respectively). This revelation demonstrated a possible scenario that the early depletion or degradation of PQD in the central region due to the harsh testing conditions. The remote film-type devices showed a similar situation but with a much lesser degree. The broadening of FWHM and the blue shift in the wavelength are certainly affected by the heat in the package. A comparison between $\mathrm{BN}-0$ and other samples in terms of a lifetime can be a very good example. Without the $\mathrm{BN}$ mixture, the heat dissipation could be worse and could cause the rapid degradation of PL of PQD and also the fast broadening of the linewidth in the optical spectrum.

3.3. TRPL. Time-resolved photoluminescence (TRPL) can be an indicator of the PQD's internal illumination capability. A comparison experiment was performed between before and

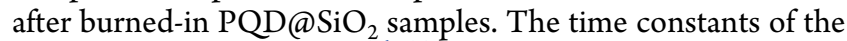
traces can be expressed as ${ }^{26-28}$

$$
I(t)=A+B_{1} \mathrm{e}^{-t / \tau_{1}}+B_{2} \mathrm{e}^{-t / \tau_{2}}+B_{3} \mathrm{e}^{-t / \tau_{3}}
$$

The multiple decaying functions can provide us some insights into the radiative transition of the quantum dots before and after the aging processes. As shown in Figure 7a, the TRPL trace of fresh PQD $@ \mathrm{SiO}_{2}$ has a longer $\tau_{1}$ (3.6 ns) than the burnt one $(1.59 \mathrm{~ns})$. A reduction in the decaying time constant can be explained as the increasing portion of the nonradiative recombination inside quantum dots. ${ }^{28,29}$
3.4. Wavelength Shift. While the remote-type package can help with the lifetime of the PQD film, the blue shift of the emission wavelength is significant and cannot be overlooked. Previous research showed that the continuous blue shift under direct laser illumination for several tens of seconds. ${ }^{30}$ In the past, many possible reasons have been discussed about the blue shift of nanocrystals. In most cases, oxidation can be a very plausible reason because of the abundance of oxygen during the quantum dot device operation. ${ }^{31}$ Other labs found that humidity can enhance the formation of smaller perovskite grains in the larger ones. ${ }^{32}$ However, after the aforementioned characterization, we found that it is not very easy to get a definitive answer to our situation. It has been recognized that the emission spectrum can usually reflect the size distribution of the nanocrystals. ${ }^{33}$ With optical spectra, one can deduce the possible size distribution of these nanocrystals, and we can extract useful information about this QD ensemble. From the previous section, our PQD@SiO 2 can maintain their size and illumination efficiency under the on-shelf test, and this result proves that our nanocrystals are naturally stable when there is no external stimulant that alters the surrounding environment of PQD greatly. However, the continuous and prolonged aging process did change the PQD's emission wavelength progressively. Even when the intensity is stable, the peak still shifts. On the other hand, both the crystallography (XRD) and chemical binding (FTIR) data seem to be the same between fresh and aged samples. There might be several explanations we can consider: (A) the formation of $\mathrm{PbBr}_{2}$ in the outer shell, (B) the internal fissure of the PQD, and (C) the shape change of the PQD. In the following, we will explain our reasoning and findings. 

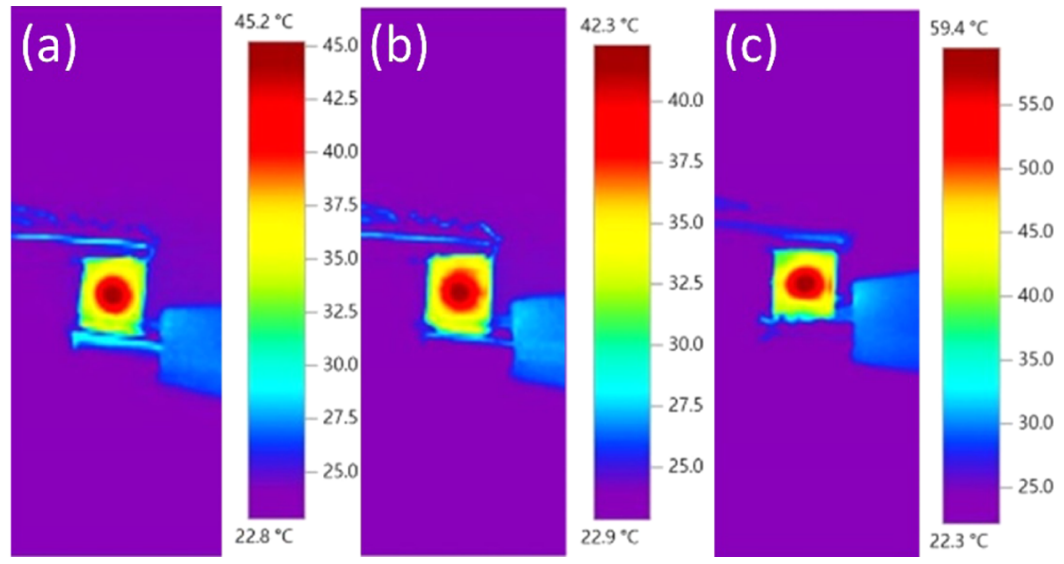

Figure 8. Thermal images of (a) a remote film-type device with $\mathrm{BN}=0 \mathrm{mg}$ at 1083th hour, and the max. temperature is $45.2^{\circ} \mathrm{C}$. (b) Remote filmtype device with $\mathrm{BN}=2 \mathrm{mg}$ at the 1126th hour, and the max. temperature is $42.3{ }^{\circ} \mathrm{C}$. (c) In-chip-type device with $\mathrm{BN}=0 \mathrm{mg}$ at the 1066 th hour, and the max. temperature is $59.4^{\circ} \mathrm{C}$.
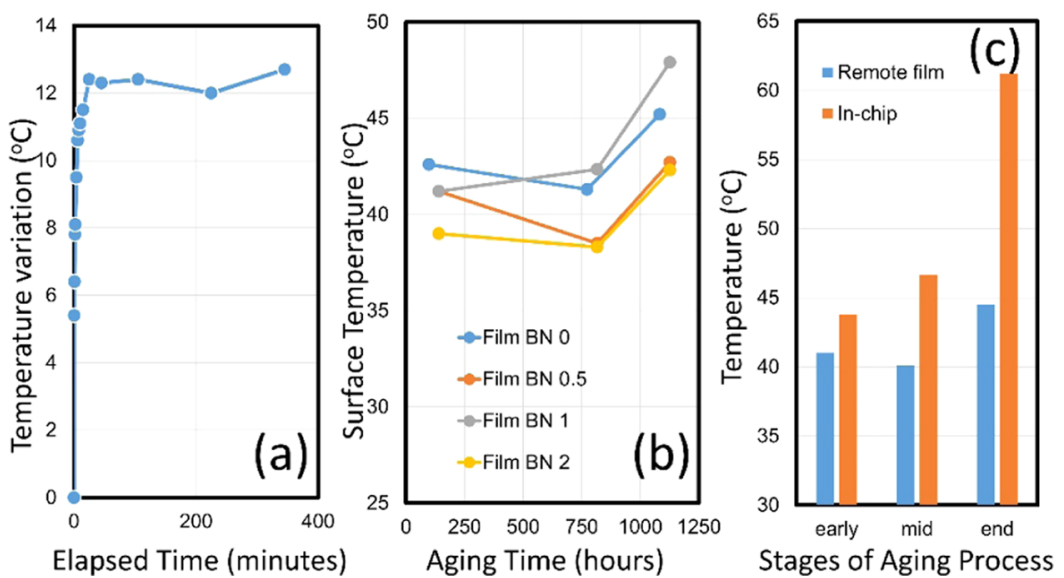

Figure 9. (a) Transient temperature variation in a $5 \mathrm{~h}$ experiment after current injection started. (b) Long-term surface temperature of our remote film devices under tests. (c) Average temperatures of remote film samples and in-chip ones at different stages of the aging tests.

While $\mathrm{PbBr}_{2}$ is one of the chemicals to form the PQD, it is also possible that the $\mathrm{PQD}$ can be decomposed and return back to $\mathrm{PbBr}_{2}{ }^{34}$ The heat and external photons provide a hotbed leading to this path. Once the outer shell is transformed to $\mathrm{PbBr}_{2}$, it loses its illumination capability. Thus, the effective size of the quantum dot reduces and causes the blue shift of the wavelength. However, the XRD result before and after aging did not show many differences, especially for the $\mathrm{PbBr}_{2}$ peak locations. Another possibility is to have a transformation between $\mathrm{Cs}_{4} \mathrm{PbBr}_{6}$ and $\mathrm{Cs} \mathrm{PbBr}_{3}$ due to various external influences. ${ }^{35,36}$ Either from the result of $\mathrm{PbBr}_{2}$ addition or $\mathrm{H}_{2} \mathrm{O}$ desorption, the transformation of the $\mathrm{CsBr}-\mathrm{PbBr}_{2}$ composite system can provide either highly luminous $\mathrm{CsPbBr}_{3}$ or photonic-inactive $\mathrm{Cs}_{4} \mathrm{PbBr}_{6}$.

The second possibility is the internal fissure of the PQD, meaning that it might possible that the PQD broke up during the aging test. When the dot breaks, the size becomes smaller and the wavelength can move toward the higher-energy side. Because this breakage is random, the linewidth of the spectrum, which is the distribution of the size of the PQD ensemble, can be widened. A similar phenomenon was reported before in $\mathrm{PQD},{ }^{29}$ and it was attributed to the Ostwald ripening of the PQD during the storage. ${ }^{29,37}$ A significant tail at the shorter-wavelength side, as shown in Figure $7 \mathrm{~b}$, can be an indicator of this situation. (The detailed spectra can be found in Figures S3 and S4 of the Supporting Information.)

To estimate the quantum dot size, the peak emission wavelength is used for the effective transition energy due to quantum confinement. Our calculation follows the effective mass theory, ${ }^{4,38}$ and the expression is

$$
E_{\text {PL_peak }}=E_{\mathrm{g}}+\frac{\hbar^{2} \pi^{2}}{2 r^{2}}\left(\frac{1}{m_{\mathrm{e}}^{*}}+\frac{1}{m_{\mathrm{h}}^{*}}\right)-E_{\text {exciton }}
$$

where $E_{\mathrm{g}}$ is the band gap of $\mathrm{CsPbr}_{3}$ (equal to $2.32 \mathrm{eV}$ ), $m_{\mathrm{e}}^{*}$ and $m_{\mathrm{h}}^{*}$ are the effective mass of $\mathrm{CsPbBr}, r$ is the average size of the dot, and $E_{\text {exciton }}$ is the binding energy of the exciton in this material (equal to $40 \mathrm{meV}){ }^{4}$ Figure $7 \mathrm{c}$ shows the calculated dot size of the corresponding emission peaks during the aging process. Both samples started with similar size distribution of dots and ended up with very different size distribution at the end of 2700 plus burn-in hours. The final estimated sizes are $4.83 \mathrm{~nm}$ (no BN) vs $5.48 \mathrm{~nm}$ (with BN 2 $\mathrm{mg})$.

The third hypothesis is the shape change (sintering or morphological change) of the dots. In the previous work, the researcher reported the elongated shape of the dots after aging, and this led to the blue shift of the wavelength. ${ }^{39}$ The sintering process could happen due to the extreme heat and UV excitation conditions that were imposed on these nanocryst- 
als. $^{39}$ In our experiment, by comparison of transmission electron microscopy (TEM) pictures of limited samples, we could have a slight increase of elongated dots after the aging process. As shown in Figure $7 d$, if we plot the length ratio of dots in the $x$-direction over that in the $y$-direction, the fresh sample has a more concentrated distribution around 1, while the samples after aging have a wider range of the size ratio. The TEM pictures are provided in Figure S6 of the Supporting Information.

From these observations, we could say that the continuous change of wavelength is an important subject to be solved in this type of PQDs because it did change the color provided by this LED. To summarize what we found in various experiments and theoretical analyses, two possible scenarios can be proposed: the reduction in the size of the quantum dot core and the change of the shape of quantum dots. Both of them can be supported from the past literature works, TEM pictures (in Figure S6), and the broadening of the optical spectra of the aged quantum dots. Meanwhile, other factors can be also the cause of this phenomenon. More investigation will be necessary before the conclusion can be drawn.

3.5. Thermal Properties of Samples. The thermal characteristics of these samples are one of the important parameters that we need to find out. To do that, the surface temperature of the sample is an easy way to start. Figure $8 \mathrm{a}-\mathrm{c}$ shows the thermal images taken at the later stage of this stressed aging process ( $>1000 \mathrm{~h}$ of continuous tests). The inchip sample without $\mathrm{BN}$ particles showed the highest surface temperature, while the other two were about $15^{\circ}$ lower.

Another important thing is to observe the temperature variation versus time. There are two kinds of data: short-term and long-term records. First, we can take a look on the shortterm response or transient response. In Figure 9a, we measure the temperature variation versus time right after the current is turned on. The increase of package temperature can be stabilized within the first $15 \mathrm{~min}$, and after that, the variation would be small (within $1 \%$ of the average temperature). This number gave us a guideline when measuring the surface temperature of these samples. Then, the long-term effect on the surface temperature is presented in Figure 9b, which demonstrates a slow but increasing trend of the temperature across aging time, from the early stage to the late stage of aging. The possible reason is due to the degradation of the quantum dots, and this situation leads to increasing nonradiative recombination at the later stage of the aging test. Also, in general, the samples with $\mathrm{BN}$ particles showed less heating on the surface. If the average temperatures are put together, the in-chip samples have much higher surface temperatures than their remote film counterparts in every stage of the aging process, as shown in Figure 9c. From these results, we can see that the gap between in-chip and remote grows when the quantum dots deteriorate (this gap can be as large as $16^{\circ}$ ), and the differences in temperature between with and without $\mathrm{BN}$ samples are about $3^{\circ}$.

To simulate these phenomena, we built a COMSOL model with similar physical sizes to the actual devices and using a LED chip at the center as the heat source. A $1 \mathrm{~W}$ of heat energy was set in the chip location. As shown in Figure 10a, the direct contact of the PQD composite with the LED surface could induce a much higher temperature inside the QD layer and eventually lead to fast degradation of the quantum dots. Meanwhile, the temperature distribution of the remote film device was more uniform than the in-chip device and as high as

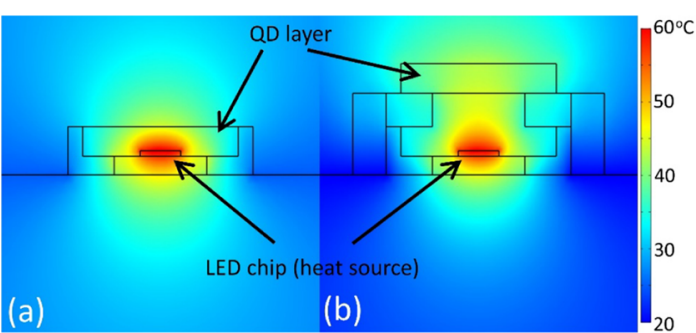

Figure 10. COMSOL calculation of temperature distribution of (a) in-chip sample and (b) remote film sample.

$14^{\circ}$ of temperature difference could be seen at the bottom of the $\mathrm{QD}$ layer in Figure 10a,b. Although the surface temperature was not that much different $\left(3-5^{\circ}\right.$ according to different physical settings), the interior temperatures of the inchip and remote film samples could be a decisive factor that causes the different aging results we obtained in this study.

\section{CONCLUSIONS}

In conclusion, we demonstrated highly stable hybrid perovskite quantum-dot LEDs with boron nitride nanoparticles incorporated in the package. While the PQD@SiO 2 powders can be stored in air for more than 250 days without significant degradation, the long and continuous operation in the LED package is still proved to be difficult. In this study, after $2500 \mathrm{~h}$ of aging, the intensity of PQD can be stabilized, while its wavelength of emission blue-shifts continuously. From the material characterization, we see very few pieces of evidence about chemical changes, but from the optical measurement, a significant change in the PQD size distribution can be observed. Three hypotheses can be proposed but need more scrutiny. Once solved, there is no doubt that inorganic perovskite quantum dots are suitable for the future adaptation of the solid-state lighting devices that involve nanoscale fluorophores.

\section{EXPERIMENTAL SECTION}

5.1. Perovskite Quantum Dot Sample Preparation. The colloidal $\mathrm{Cs}_{\mathrm{PbBr}}$ PQDs in this study were prepared in two steps: one is the Cs-oleate precursor material preparation, and the other is the synthesis of $\mathrm{CsPbBr}_{3} \cdot{ }^{4040}$ For the Cs-oleate step, we put $0.814 \mathrm{~g}$ of $\mathrm{Cs}_{2} \mathrm{CO}_{3}, 2.5 \mathrm{~mL}$ of oleic acid (OA, $90 \%$ ), and $40 \mathrm{~mL}$ of 1 -octadencene (ODE, 90\%) into a 100 $\mathrm{mL}$ three-necked bottle and mix them fully. The mixture is heated to $120^{\circ} \mathrm{C}$ in vacuum for $1 \mathrm{~h}$, followed by heating at 150 ${ }^{\circ} \mathrm{C}$ in $\mathrm{N}_{2}$ until all $\mathrm{Cs}_{2} \mathrm{CO}_{3}$ completely reacts with $\mathrm{OA}$. For the step of synthesizing $\mathrm{CsPbBr}_{3}$, we put $0.752 \mathrm{mmol}$ lead(II) bromide $\left(\mathrm{PbBr}_{2}, 99 \%\right)$ and $20 \mathrm{~mL}$ of ODE into a $100 \mathrm{~mL}$ three-necked bottle and mix them fully and then the mixture is heated at $120^{\circ} \mathrm{C}$ in vacuum conditions for $1 \mathrm{~h}$. Then, we heat them at $120{ }^{\circ} \mathrm{C}$ with $\mathrm{N}_{2}$, inject $2 \mathrm{~mL}$ of $\mathrm{OA}$, and $2 \mathrm{~mL}$ of oleylamine (OAm, 80-90\%) until $\mathrm{PbBr}_{2}$ reacts with $\mathrm{OA}$ and OAm totally. Finally, we increase the temperature to $180{ }^{\circ} \mathrm{C}$ and inject $1.6 \mathrm{~mL}$ of Cs-oleate solution wait for $5 \mathrm{~s}$ then cooling it. After the synthesis of nanocrystals (NCs) is finished, (3-aminopropyl)triethoxysilane (APTES) would then be added to the PQD solution and stirred for $2 \mathrm{~h}$ and then tetramethoxysilane (TMOS) would be added to further stirred for another $38 \mathrm{~h}$. The $-\mathrm{NH}_{2}$ functional group of APTES is used to silylize the surface of $\mathrm{PQD}$, and the hydrolysis reaction of TMOS can lead to the formation of $\mathrm{SiO}_{2}$ around the 
quantum dots. After the reaction is completed, the solution is centrifuged at $9000 \mathrm{rpm}$ and then evacuated to extract the $\mathrm{PQD} @ \mathrm{SiO}_{2}$ powder. Figure 11 shows the dried $\mathrm{PQD} @ \mathrm{SiO}_{2}$ powder under a UV lamp, and Figure $11 \mathrm{~b}$ shows the transmission electron microscopy (TEM) picture of a $\mathrm{Cs} \mathrm{PbBr}_{3}$ NC.

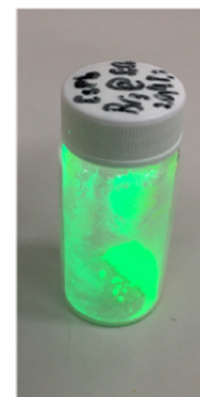

(a)

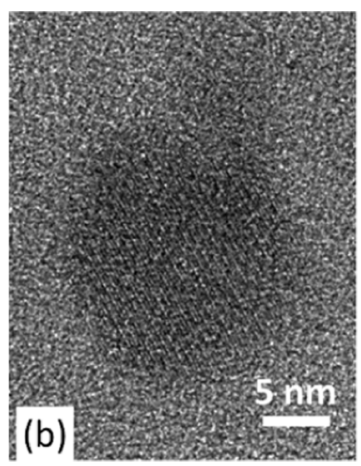

Figure 11. (a) $\mathrm{PQD} @ \mathrm{SiO}_{2}$ powder under $\mathrm{UV}$ and (b) $\mathrm{PQD} @ \mathrm{SiO}_{2}$ by TEM.

Next, we used the dried powder of green 530 nm PQD@ $\mathrm{SiO}_{2}$ and the powder was mixed with PDMS at the weight ratio of $1: 9$ to form the filling substances in the experiments. The pumping source in this study was the UV LED chip made by Epistar Corporation. The UV chips were first driven at $250 \mathrm{~mA}$ for at least $24 \mathrm{~h}$ to stabilize their output power. Since the normal bias current level was lower than $250 \mathrm{~mA}$, this burn-in step could ensure a constant photoexcitation intensity in the following aging process. There were two different structures considered in our study. In the first structure, the PQD@SiO powder was mixed uniformly with PDMS slurry and placed around the UV LED chip, and this type of device was named "in-chip" type. For the second structure, called the "remote film” type, the PQD@SiO 2 + PDMS film was first mixed, dried, and then placed on the copper tape with a $2.5 \mathrm{~mm}$ diameter hole at the center and attached on top of the 5070 lead frame. One of the important steps in our manufacturing of these remote films was the molding step. The PQD + PDMS mixture was filled into an empty 5070 lead frame and baked. Then, the film would be peeled out after it became solid. With a precise control on the weight of the PQD + PDMS mixture and the molding step, an approximate shape (height and width) can be maintained from device to device. There were no other filling materials in the remote film style package, and the PQD film was suspended above the UV chip. The purpose of this experimental design was to verify that our remote film package can further improve the reliability of the PQD device even under continuously stressed aging situation. Using the in-chip style package could benchmark our PQDs with the traditional method, and the on-shelf lifetest in the next section could verify our good material quality. ${ }^{17,41-43}$ For both types of samples, we placed the composites on the holder and then cured them at $70{ }^{\circ} \mathrm{C}$ to solidify the slurry. To improve thermal dissipation, PQD@SiO ${ }_{2}+\mathrm{PDMS}$ composites were mixed with certain weights of hexagonal boron nitride (h-BN) nanoparticles and reference samples without $\mathrm{BN}$ particles were also made at the same time. The h-BN powder was purchased from Sigma-Aldrich, and its average particle size was $1 \mu \mathrm{m}$ (product number 255475-50 G). For the in-chip structure, we had two samples: one was $\mathrm{BN}-0 \mathrm{mg}$ (no $\mathrm{BN}$ ), and the other was $\mathrm{BN}-2$ $\mathrm{mg}$ (with $2 \mathrm{mg}$ of $\mathrm{BN}$ powder added). For the remote film structure, four samples, BN-0 mg, BN-0.5 mg, BN-1 mg, and $\mathrm{BN}-2 \mathrm{mg}$, were produced according to the weights of $\mathrm{BN}$ particles inside the PQD@SiO $2+$ PDMS composites. Figure $12 \mathrm{a}, \mathrm{b}$ shows the process flow and the packaged structures of the two types of devices.

5.2. Measurement Methods. The devices were measured in an integrated sphere coated with the $\mathrm{BaSO}_{4}$ material. Keithley 2400 source meters are used to supply suitable currents to the LEDs. The photoluminescence of the thin films, which was analyzed by the on-shelf storage test, was measured by a Princeton Instruments Acton 2150 system with calibrated samples for the long-term stability test. Fourier transform infrared (FTIR) spectroscopy was performed by a Thermo-Fisher Scientific Nicolet iS10 FTIR spectrometer to determine any possible variation in the chemical bonding before and after PQD-aging tests.

After the fabrication of six devices was finished, these devices were driven by a continuous current at room temperature and the electrical currents were provided by a Keithley 2400 source meter. In this process, their electrical and optical properties were extracted from the integrated sphere system. The lifetime tests were conducted in the constant current mode with the
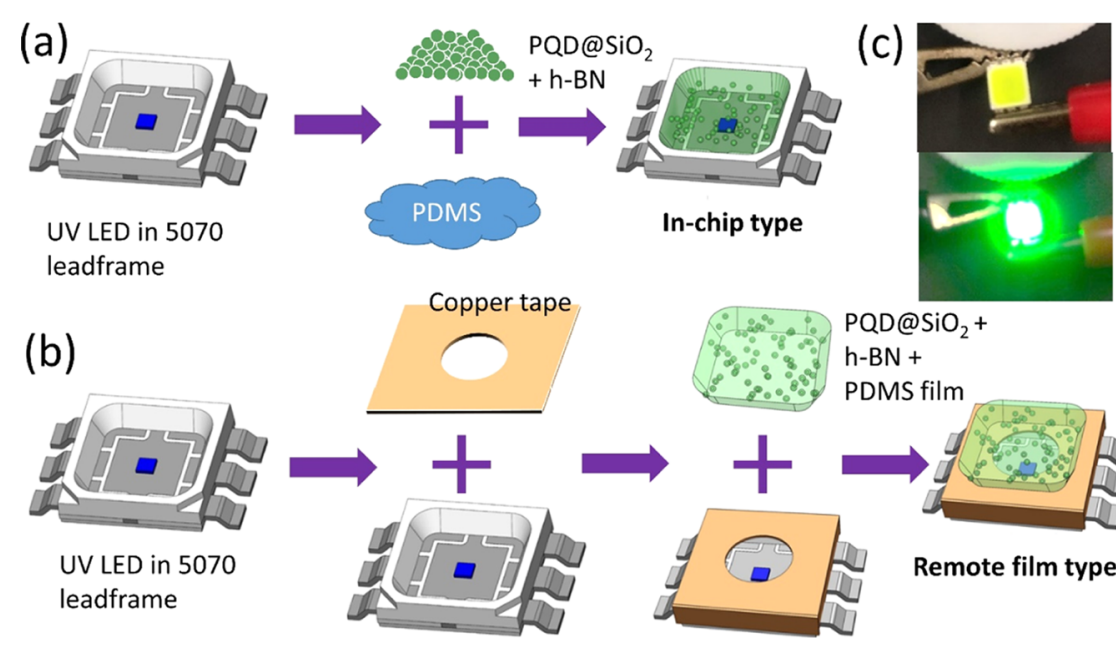

$\mathrm{PQD} @ \mathrm{SiO}_{2}+$

$\mathrm{h}-\mathrm{BN}+$

PDMS film

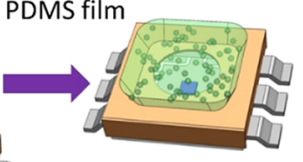

Remote film type

Figure 12. Process flows for (a) in-chip devices and (b) remote film devices. (c) On-chip device with current off (up) and on (down). 
consideration of the constant photonic pumping intensity of 1 $\mathrm{W} / \mathrm{cm}^{2}$. Figure $12 \mathrm{c}$ shows an "in-chip" device with and without current injection.

\section{ASSOCIATED CONTENT}

\section{SI Supporting Information}

The Supporting Information is available free of charge at https://pubs.acs.org/doi/10.1021/acsomega.0c05139.

Current-dependent luminous efficiency, in-chip device aging results, spectral comparison before and after aging, TEM images of PQDs before and after burn-in, and spectral comparison of the on-shelf storage tests (PDF)

\section{AUTHOR INFORMATION}

\section{Corresponding Author}

Chien-Chung Lin - Institute of Photonic System, College of Photonics, National Chiao Tung University, Tainan 71150, Taiwan; 이이.org/0000-0001-8656-1922;

Email: chienchunglin@faculty.nctu.edu.tw

\section{Authors}

Shun-Chieh Hsu - Institute of Photonic System, College of Photonics, National Chiao Tung University, Tainan 71150, Taiwan

Yu-Ming Huang - Institute of Photonic System, College of Photonics, National Chiao Tung University, Tainan 71150, Taiwan

Chung-Ping Huang - Institute of Photonic System, College of Photonics, National Chiao Tung University, Tainan 71150, Taiwan

Ting-Yu Lee - Institute of Photonic System, College of Photonics, National Chiao Tung University, Tainan 71150, Taiwan

Yu-Yun Cho - Institute of Photonic System, College of Photonics, National Chiao Tung University, Tainan 71150, Taiwan

Yin-Hsin Liu - Department of Applied Chemistry, National Chiao Tung University, Hsinchu 30010, Taiwan

Arumugam Manikandan - Department of Material Science and Engineering, National Tsing Hua University, Hsinchu 30013, Taiwan, ROC; (1) orcid.org/0000-0001-7284-2002

Yu-Lun Chueh - Department of Material Science and Engineering, National Tsing Hua University, Hsinchu 30013, Taiwan, ROC; 이이이.0rg/0000-0002-0155-9987

Teng-Ming Chen - Department of Applied Chemistry, National Chiao Tung University, Hsinchu 30010, Taiwan; ○ orcid.org/0000-0002-8773-7720

Hao-Chung Kuo - Department of Photonics and Graduate Institute of Electro-Optical Engineering, College of Electrical and Computer Engineering, National Chiao Tung University, Hsinchu 30010, Taiwan

Complete contact information is available at:

https://pubs.acs.org/10.1021/acsomega.0c05139

\section{Notes}

The authors declare no competing financial interest.

\section{ACKNOWLEDGMENTS}

The authors thank the financial support from MOST of Taiwan under contract no. MOST-107-2221-E-009-114-MY3.

\section{ABBREVIATIONS USED}

PQD, perovskite quantum dot; NC, nanocrystal; TRPL, timeresolved photoluminescence

\section{REFERENCES}

(1) Zhao, X.; Ng, J. D. A.; Friend, R. H.; Tan, Z.-K. Opportunities and Challenges in Perovskite Light-Emitting Devices. ACS Photonics 2018, 5, 3866-3875.

(2) Murphy, C. J. Peer Reviewed: Optical Sensing with Quantum Dots. Anal. Chem. 2002, 74, 520 A-526A.

(3) Nogay, G.; Sahli, F.; Werner, J.; Monnard, R.; Boccard, M.; Despeisse, M.; Haug, F. J.; Jeangros, Q.; Ingenito, A.; Ballif, C. 25.1\%Efficient Monolithic Perovskite/Silicon Tandem Solar Cell Based on a p-type Monocrystalline Textured Silicon Wafer and High-Temperature Passivating Contacts. ACS Energy Lett. 2019, 4, 844-845.

(4) Protesescu, L.; Yakunin, S.; Bodnarchuk, M. I.; Krieg, F.; Caputo, R.; Hendon, C. H.; Yang, R. X.; Walsh, A.; Kovalenko, M. V. Nanocrystals of Cesium Lead Halide Perovskites $\left(\mathrm{CsPbX}_{3}, \mathrm{X}=\mathrm{Cl}, \mathrm{Br}\right.$ and I): Novel Optoelectronic Materials Showing Bright Emission with Wide Color Gamut. Nano Lett. 2015, 15, 3692-3696.

(5) Fang, X.; Zhang, K.; Li, Y.; Yao, L.; Zhang, Y.; Wang, Y.; Zhai, W.; Tao, L.; Du, H.; Ran, G. Effect of excess $\mathrm{PbBr} 2$ on photoluminescence spectra of $\mathrm{CH} 3 \mathrm{NH} 3 \mathrm{PbBr} 3$ perovskite particles at room temperature. Appl. Phys. Lett. 2016, 108, No. 071109.

(6) Kwak, J.; Bae, W. K.; Lee, D.; Park, I.; Lim, J.; Park, M.; Cho, H.; Woo, H.; Yoon, D. Y.; Char, K.; Lee, S.; Lee, C. Bright and Efficient Full-Color Colloidal Quantum Dot Light-Emitting Diodes Using an Inverted Device Structure. Nano Lett. 2012, 12, 2362-2366.

(7) Liang, P.; Zhang, P.; Pan, A.; Yan, K.; Zhu, Y.; Yang, M.; He, L. Unusual Stability and Temperature-Dependent Properties of Highly Emissive $\mathrm{CsPbBr}_{3}$ Perovskite Nanocrystals Obtained from in Situ Crystallization in Poly(vinylidene difluoride). ACS Appl. Mater. Interfaces 2019, 11, 22786-22793.

(8) Yuan, S.; Chen, D.; Li, X.; Zhong, J.; Xu, X. In Situ Crystallization Synthesis of $\mathrm{CsPbBr} 3$ Perovskite Quantum DotEmbedded Glasses with Improved Stability for Solid-State Lighting and Random Upconverted Lasing. ACS Appl. Mater. Interfaces 2018 10, 18918-18926.

(9) Li, Y.; Lv, Y.; Guo, Z.; Dong, L.; Zheng, J.; Chai, C.; Chen, N.; Lu, Y.; Chen, C. One-Step Preparation of Long-Term Stable and Flexible $\mathrm{CsPbBr} \mathrm{Pb}_{3}$ Perovskite Quantum Dots/Ethylene Vinyl Acetate Copolymer Composite Films for White Light-Emitting Diodes. ACS Appl. Mater. Interfaces 2018, 10, 15888-15894.

(10) Xu, L.; Chen, J.; Song, J.; Li, J.; Xue, J.; Dong, Y.; Cai, B.; Shan, Q.; Han, B.; Zeng, H. Double-Protected All-Inorganic Perovskite Nanocrystals by Crystalline Matrix and Silica for Triple-Modal AntiCounterfeiting Codes. ACS Appl. Mater. Interfaces 2017, 9, 2655626564.

(11) Zhang, J.; Jiang, P.; Wang, Y.; Liu, X.; Ma, J.; Tu, G. In Situ Synthesis of Ultrastable CsPbBr ${ }_{3}$ Perovskite Nanocrystals Coated with Polyimide in a CSTR System. ACS Appl. Mater. Interfaces 2020, 12, 3080-3085.

(12) Lv, W.; Li, L.; Xu, M.; Hong, J.; Tang, X.; Xu, L.; Wu, Y.; Zhu, R.; Chen, R.; Huang, W. Improving the Stability of Metal Halide Perovskite Quantum Dots by Encapsulation. Adv. Mater. 2019, 31, No. 1900682

(13) Sun, C.; Zhang, Y.; Ruan, C.; Yin, C.; Wang, X.; Wang, Y.; Yu, W. W. Efficient and Stable White LEDs with Silica-Coated Inorganic Perovskite Quantum Dots. Adv. Mater. 2016, 28, 10088-10094.

(14) Xie, B.; Liu, H.; Hu, R.; Wang, C.; Hao, J.; Wang, K.; Luo, X. Targeting Cooling for Quantum Dots in White QDs-LEDs by Hexagonal Boron Nitride Platelets with Electrostatic Bonding. Adv. Funct. Mater. 2018, 28, No. 1801407.

(15) Lin, C.-C.; Liu, W.-L.; Hsieh, C.-Y. Scalar scattering model of highly textured transparent conducting oxide. J. Appl. Phys. 2011, 109, No. 014508.

(16) Hsu, S. C.; Chen, Y. H.; Tu, Z. Y.; Han, H. V.; Lin, S. L.; Chen, T. M.; Kuo, H. C.; Lin, C. C. Highly Stable and Efficient Hybrid 
Quantum Dot Light-Emitting Diodes. IEEE Photonics J. 2015, 7, 110 .

(17) Hsu, S. C.; Ke, L. A.; Lin, H. C.; Chen, T. M.; Lin, H. Y.; Chen, Y. Z.; Chueh, Y. L.; Kuo, H. C.; Lin, C. C. Fabrication of a Highly Stable White Light-Emitting Diode With Multiple-Layer Colloidal Quantum Dots. IEEE J. Sel. Top. Quantum Electron. 2017, 23, 1-9.

(18) Gao, G.; Mathkar, A.; Martins, E. P.; Galvão, D. S.; Gao, D.; da Silva Autreto, P. A.; Sun, C.; Cai, L.; Ajayan, P. M. Designing nanoscaled hybrids from atomic layered boron nitride with silver nanoparticle deposition. J. Mater. Chem. A 2014, 2, 3148-3154.

(19) Kuo, H.-C.; Hung, C.-W.; Chen, H.-C.; Chen, K.-J.; Wang, C.H.; Sher, C.-W.; Yeh, C.-C.; Lin, C.-C.; Chen, C.-H.; Cheng, Y.-J. Patterned structure of REMOTE PHOSPHOR for phosphorconverted white LEDs. Opt. Express 2011, 19, A930-A936.

(20) Kim, Y.; Yassitepe, E.; Voznyy, O.; Comin, R.; Walters, G.; Gong, X.; Kanjanaboos, P.; Nogueira, A. F.; Sargent, E. H. Efficient Luminescence from Perovskite Quantum Dot Solids. ACS Appl. Mater. Interfaces 2015, 7, 25007-25013.

(21) Zhong, Q.; Cao, M.; Hu, H.; Yang, D.; Chen, M.; Li, P.; Wu, L.; Zhang, Q. One-Pot Synthesis of Highly Stable CsPbBr ${ }_{3} @ \mathrm{SiO}_{2}$ Core-Shell Nanoparticles. ACS Nano 2018, 12, 8579-8587.

(22) Uddin, M. A.; Calabro, R. L.; Kim, D.-Y.; Graham, K. R. Halide exchange and surface modification of metal halide perovskite nanocrystals with alkyltrichlorosilanes. Nanoscale 2018, 10, 1691916927.

(23) Maria Chong, A. S.; Zhao, X. S. Functionalization of SBA-15 with APTES and Characterization of Functionalized Materials. J. Phys. Chem. B 2003, 107, 12650-12657.

(24) Wang, W.; Wang, D.; Fang, F.; Wang, S.; Xu, G.; Zhang, T. $\mathrm{CsPbBr} 3 / \mathrm{Cs} 4 \mathrm{PbBr} 6$ Nanocomposites: Formation Mechanism, Largescale and Green Synthesis, and Application in White Light-Emitting Diodes. Cryst. Growth Des. 2018, 18, 6133-6141.

(25) Shi, Z.; Li, S.; Li, Y.; Ji, H.; Li, X.; Wu, D.; Xu, T.; Chen, Y.; Tian, Y.; Zhang, Y.; Shan, C.; Du, G. Strategy of Solution-Processed All-Inorganic Heterostructure for Humidity/Temperature-Stable Perovskite Quantum Dot Light-Emitting Diodes. ACS Nano 2018, 12, 1462-1472.

(26) Zhou, L.; Yu, K.; Yang, F.; Cong, H.; Wang, N.; Zheng, J.; Zuo, Y.; Li, C.; Cheng, B.; Wang, Q. Insight into the effect of ligandexchange on colloidal $\mathrm{Cs} \mathrm{PbBr} 3$ perovskite quantum dot/mesoporous$\mathrm{TiO} 2$ composite-based photodetectors: much faster electron injection. J. Mater. Chem. C 2017, 5, 6224-6233.

(27) Cao, P.; Yang, B.; Geng, Y.; Cao, Y.; Wang, L.; Zheng, F.; Zou, J. Heat-Stable and Moisture-Proof CsPbBr3 Quantum Dots Under Dual Protection: Coordination of Non-Crystalline $\mathrm{SiO} 2$ and Crystalline h-BN Nanosheets. ECS J. Solid State Sci. Technol. 2020, 9, No. 046001.

(28) An, R.; Zhang, F.; Zou, X.; Tang, Y.; Liang, M.; Oshchapovskyy, I.; Liu, Y.; Honarfar, A.; Zhong, Y.; Li, C.; Geng, H.; Chen, J.; Canton, S. E.; Pullerits, T.; Zheng, K. Photostability and Photodegradation Processes in Colloidal CsPbI3 Perovskite Quantum Dots. ACS Appl. Mater. Interfaces 2018, 10, 39222-39227.

(29) Xie, Q.; Wu, D.; Wang, X.; Li, Y.; Fang, F.; Wang, Z.; Ma, Y.; Su, M.; Peng, S.; Liu, H.; Wang, K.; Sun, X. W. Branched capping ligands improve the stability of cesium lead halide ( $\mathrm{CsPbBr} 3)$ perovskite quantum dots. J. Mater. Chem. C 2019, 7, 11251-11257.

(30) Swarnkar, A.; Chulliyil, R.; Ravi, V. K.; Irfanullah, M.; Chowdhury, A.; Nag, A. Colloidal CsPbBr3 Perovskite Nanocrystals: Luminescence beyond Traditional Quantum Dots. Angew. Chem., Int. Ed. 2015, 54, 15424-15428.

(31) Foos, E. E. The Complex Interaction of Spectroscopic Shifts and Electronic Properties in Semiconductor Nanocrystal Films. J. Phys. Chem. Lett. 2013, 4, 625-632.

(32) Di Girolamo, D.; Dar, M. I.; Dini, D.; Gontrani, L.; Caminiti, R.; Mattoni, A.; Graetzel, M.; Meloni, S. Dual effect of humidity on cesium lead bromide: enhancement and degradation of perovskite films. J. Mater. Chem. A 2019, 7, 12292-12302.

(33) Crooker, S. A.; Hollingsworth, J. A.; Tretiak, S.; Klimov, V. I. Spectrally Resolved Dynamics of Energy Transfer in Quantum-Dot
Assemblies: Towards Engineered Energy Flows in Artificial Materials. Phys. Rev. Lett. 2002, 89, No. 186802.

(34) Akbali, B.; Topcu, G.; Guner, T.; Ozcan, M.; Demir, M. M.; Sahin, H. CsPbBr3 perovskites: Theoretical and experimental investigation on water-assisted transition from nanowire formation to degradation. Phys. Rev. Mater. 2018, 2, No. 034601.

(35) Akkerman, Q. A.; Park, S.; Radicchi, E.; Nunzi, F.; Mosconi, E.; De Angelis, F.; Brescia, R.; Rastogi, P.; Prato, M.; Manna, L. Nearly Monodisperse Insulator $\mathrm{Cs}_{4} \mathrm{PbX}_{6}(\mathrm{X}=\mathrm{Cl}, \mathrm{Br}, \mathrm{I})$ Nanocrystals, Their Mixed Halide Compositions, and Their Transformation into CsPbX3 Nanocrystals. Nano Lett. 2017, 17, 1924-1930.

(36) Liu, M.; Zhao, J.; Luo, Z.; Sun, Z.; Pan, N.; Ding, H.; Wang, X. Unveiling Solvent-Related Effect on Phase Transformations in CsBr$\mathrm{PbBr}_{2}$ System: Coordination and Ratio of Precursors. Chem. Mater. 2018, 30, 5846-5852.

(37) Koolyk, M.; Amgar, D.; Aharon, S.; Etgar, L. Kinetics of cesium lead halide perovskite nanoparticle growth; focusing and de-focusing of size distribution. Nanoscale 2016, 8, 6403-6409.

(38) Kim, Y.-H.; Wolf, C.; Kim, Y.-T.; Cho, H.; Kwon, W.; Do, S.; Sadhanala, A.; Park, C. G.; Rhee, S.-W.; Im, S. H.; Friend, R. H.; Lee, T.-W. Highly Efficient Light-Emitting Diodes of Colloidal MetalHalide Perovskite Nanocrystals beyond Quantum Size. ACS Nano 2017, 11, 6586-6593.

(39) Ihly, R.; Tolentino, J.; Liu, Y.; Gibbs, M.; Law, M. The Photothermal Stability of PbS Quantum Dot Solids. ACS Nano 2011, 5, 8175-8186.

(40) Chung, M.-T. Characterizations of Derivatives of All-Inorganic Perovskite Quantum Dots and Their Applications in White LEDs and Color-Enhancement Films. Master's Thesis, National Chiao Tung University, Hsinchu, Taiwan, 2017.

(41) Morrell, M. V.; He, X.; Luo, G.; Thind, A. S.; White, T. A.; Hachtel, J. A.; Borisevich, A. Y.; Idrobo, J.-C.; Mishra, R.; Xing, Y. Significantly Enhanced Emission Stability of $\mathrm{Cs} \mathrm{PbBr}_{3}$ Nanocrystals via Chemically Induced Fusion Growth for Optoelectronic Devices. ACS Appl. Nano Mater. 2018, 1, 6091-6098.

(42) Li, Z.; Kong, L.; Huang, S.; Li, L. Highly Luminescent and Ultrastable $\mathrm{CsPbr}_{3}$ Perovskite Quantum Dots Incorporated into a Silica/Alumina Monolith. Angew. Chem., Int. Ed. 2017, 56, 81348138.

(43) Huang, S.; Li, Z.; Wang, B.; Zhu, N.; Zhang, C.; Kong, L.; Zhang, Q.; Shan, A.; Li, L. Morphology Evolution and Degradation of $\mathrm{CsPbBr} 3$ Nanocrystals under Blue Light-Emitting Diode Illumination. ACS Appl. Mater. Interfaces 2017, 9, 7249-7258. 